\title{
SPONTANEOUS SYMMETRY BREAKING OF A HYPERBOLIC SIGMA MODEL IN THREE DIMENSIONS
}

\author{
by
}

T. Spencer \& M.R. Zirnbauer

\begin{abstract}
Non-linear sigma models that arise from the supersymmetric approach to disordered electron systems contain a non-compact bosonic sector. We study the model with target space $\mathrm{H}^{2}$, the two-hyperboloid with isometry group $\mathrm{SU}(1,1)$, and prove that in three dimensions moments of the fields are finite in the thermodynamic limit. Thus the non-compact symmetry $\mathrm{SU}(1,1)$ is spontaneously broken. The bound on moments is compatible with the presence of extended states.
\end{abstract}

Keywords: disordered electron systems, band random matrices, extended states, nonlinear sigma model, non-compact symmetric spaces, convexity methods.

\section{Introduction}

Random-matrix ensembles such as the Gaussian Unitary Ensemble (GUE) and its cousins have attracted much attention in both the physics and mathematics community because of its many connections to statistical many-body theory, integrable systems, number theory and probability. This article is motivated by the study of Gaussian matrix ensembles which incorporate spatial structure and thus are no longer mean field in character. These ensembles are sometimes called band GUE models. They have the advantage of being mathematically more tractable than say random Schrödinger operators and yet they are expected to share the same qualitative features.

About twenty-five years ago, Wegner $[\mathbf{W}, \mathbf{S}]$ introduced hyperbolic non-linear sigma models to study band GUE models and disordered electron systems with $N$ orbitals per site. In the simplest case of these sigma models the hyperbolic 'spins' indexed by lattice sites of $\mathbb{Z}^{d}$ take values in the hyperbolic plane $\mathrm{H}^{2}$ equipped with its $\mathrm{SU}(1,1)$ invariant geometry. Soon thereafter, Efetov $[\mathbf{E}]$ extended Wegner's work and introduced a class of supersymmetric non-linear sigma models.

The supersymmetric formalism has the advantage of making it possible to perform the disorder average and rigorously convert random matrices to a problem in statistical mechanics. In particular, averages of products of Green's functions become statistical 
mechanical correlation functions. The resulting problem is how to analyse such statistical mechanics systems. One of the main difficulties in this analysis is the non-compact hyperbolic symmetry identified by Wegner which naturally arises when studying spectral and transport properties of disordered systems. This paper is devoted to showing that a certain class of $S U(1,1)$ sigma models can be effectively analysed in three dimensions by using horospherical coordinates and Brascamp-Lieb inequalities.

Let $\Lambda$ be a periodic box in $\mathbb{Z}^{d}$ (centered at 0 ) with volume $|\Lambda|$ and define $R(i, j)$ with $i, j \in \Lambda$ to be the elements of a Hermitian matrix drawn from the GUE. Thus the probability density is taken to be proportional to $\exp \left(-\operatorname{Tr} R^{2}\right) \prod d R(i, j)$. Now let $J$ be a symmetric matrix with positive entries $J(i, j)$ which are small when the distance $|i-j|$ is large. Then define a band matrix $H$ with matrix elements

$$
H(i, j)=\sqrt{J(i, j)} R(i, j) .
$$

If we set

$$
J(i, j)=\left(-W^{2} \Delta+1\right)^{-1}(i, j)
$$

(with $\Delta$ the Laplacian of the lattice $\Lambda$ ), then this corresponds to a band of width $W$. Note that $J(i, j)$ has exponential decay $\mathrm{e}^{-|i-j| / W}$. Another convenient choice of $J$ is given as follows. Suppose that $\Lambda$ is tiled by identical cubes of width $W$. Then define

$$
J(i, j)= \begin{cases}J_{0} & \text { if } i \text { and } j \text { belong to the same cube, } \\ J_{1} & \text { if } i \text { and } j \text { belong to adjacent cubes, } \\ 0 & \text { otherwise. }\end{cases}
$$

In both cases the matrix elements far from the diagonal are suppressed or vanish, and now the dimension of the lattice plays an important role. One expects that $H$ has qualitatively the features of a random Schrödinger operator as we let the box $\Lambda$ approach $\mathbb{Z}^{d}$. In fact, for the infinite-volume limit and fixed $W$ we know that $H$ has pure point spectrum (and thus localization) at all energies in one dimension, and in any dimension there is localization for energies in the Lifshitz tails. Large $W$ is expected to be roughly inversely proportional to the strength $\lambda$ of the random potential. For example in one dimension the localization length is proportional to $\lambda^{-2}$ for the random Schrödinger operator, and proportional to $W^{2}$ for a band random matrix [[FM].

For dimension $d=3$, with $J$ given by (1.2) and large $W$, the average local density of states

$$
\rho(E)=\pi^{-1} \mathfrak{I m}\left\langle(H-E-\mathrm{i} \varepsilon)^{-1}(x, x)\right\rangle
$$

was studied using the supersymmetric formalism in the limit when $\varepsilon$ goes to zero. $\rho(E)$ was shown to be smooth for $E$ in an interval around zero and field correlations were proven to decay exponentially fast $[\mathbf{D}]$.

To get information about time evolution or the behavior of the eigenstates, expectations like (1.4) are not sufficient. Instead one must consider

$$
\left\langle\left|(H-E-\mathrm{i} \varepsilon)^{-1}(x, y)\right|^{2}\right\rangle
$$


and study its behavior as $\varepsilon$ goes to zero. If (1.5) remains bounded for $x=y$ and $\varepsilon|\Lambda| \rho(E)=1$, then the eigenstates near $E$ are extended in the sense that the $L^{4}$ norm of an $L^{2}$ normalized eigenstate goes to zero in the limit $|\Lambda| \rightarrow \infty$.

What makes (1.5) more difficult to analyse than (1.4) is that the absolute value squared eliminates oscillations and small denominators are felt when $\varepsilon$ is small. In fact, even in finite volume (1.4) does not diverge as $\varepsilon$ goes to zero. However, (1.5) diverges roughly like $\rho /\left(\varepsilon \xi^{d}\right)$ where $\xi$ is the length over which the eigenfunctions are extended. Roughly speaking, the hyperbolic symmetry emerges because the two Green's functions in (1.5) have energies with imaginary parts of opposite signs. Another feature of (1.5) is that the corresponding statistical mechanics model is not expected to decay rapidly in 3 dimensions but rather to exhibit a Goldstone mode so that (1.5) should behave in the limit $\varepsilon \rightarrow 0$ like $1 /|x-y|$, the Green's function of the Laplacian corresponding to diffusive time evolution.

The main purpose of this article is to analyse Wegner's non-linear sigma model (for one replica) as a component of Efetov's supersymmetric model. More precisely we study a sigma model that arises in Fyodorov's work $[\mathbf{F}]$. This model is formulated on a lattice, whereas Wegner's model emerges upon taking a continuum limit.

Roughly speaking supersymmetric models of disordered quantum systems have three sectors: the Boson-Boson, Fermion-Fermion and Boson-Fermion sectors. The BB sector has the hyperbolic symmetry; this is the sector which we study. Although the field in this sector may potentially diverge we show that for dimension $d \geq 3$, if $\varepsilon|\Lambda| \rho=1$, all moments of the field remain uniformly bounded. This is the analogue (in the sigma model approximation) of the conjectured bound on (1.5) in $d \geq 3$. The F-F sector may also be studied in the sigma model approximation, and it corresponds to a classical Heisenberg model taking values in the two-dimensional sphere. Considered on its own, this sector has no divergence because of the compactness of the target. In three dimensions, the nearest neighbor Heisenberg model has an ordered state which may be established by using infrared bounds. The main open problem which we do not address in this article is the B-F sector, which couples the other two sectors in a supersymmetric fashion. This must be understood to obtain a complete picture of the SUSY models. Nevertheless, we will see that many phenomena of interest are already reflected in the sigma model analysed in this article.

We now describe the hyperbolic sigma model which we shall analyse. In a periodic box $\Lambda \in \mathbb{Z}^{d}$ (not the $\Lambda$ of before, but the lattice of cubes that tile the original lattice), we associate to each lattice site $j \in \Lambda$ a matrix

$$
S_{j}=T_{j} \sigma_{3} T_{j}^{-1}
$$

where $T_{j}$ is subject to the conditions $T^{*} \sigma_{3} T=\sigma_{3}=\operatorname{diag}(1,-1)$ and $\operatorname{Det} T_{j}=1$. Thus $T_{j}$ belongs to $\mathrm{SU}(1,1)$, and $S_{j}$ belongs to an adjoint orbit of $\mathrm{SU}(1,1)$, which may be identified with the symmetric space $\mathrm{SU}(1,1) / \mathrm{U}(1) \cong \mathrm{H}^{2}$ where $\mathrm{U}(1)$ is the isotropy subgroup generated by $i \sigma_{3}$. 
The action or energy of a configuration $j \mapsto S_{j}$ is given by

$$
A_{\Lambda}(S, h)=\frac{1}{2} \sum_{\Lambda}^{\prime} \operatorname{Tr}\left(S_{j} S_{j^{\prime}}\right)+\frac{1}{2} h \sum_{j \in \Lambda} \operatorname{Tr}\left(\sigma_{3} S_{j}\right)
$$

where $\Sigma_{\Lambda}^{\prime}$ denotes the sum over pairs of nearest-neighbor sites of $\Lambda$, and $h>0$. Let $d \mu(S)$ denote an invariant measure on $\mathrm{SU}(1,1) / \mathrm{U}(1)$ and define

$$
Z_{\Lambda}(\beta, h)=\int \mathrm{e}^{-\beta A_{\Lambda}(S, h)} \prod_{j \in \Lambda} d \mu\left(S_{j}\right)
$$

Expectations in this model are given by

$$
Z_{\Lambda}(\beta, h)^{-1} \int F(S) \mathrm{e}^{-\beta A_{\Lambda}(S, h)} \prod_{j \in \Lambda} d \mu\left(S_{j}\right)=\langle F\rangle_{\Lambda}(\beta, h) .
$$

Note that by $M:=S \sigma_{3}=T T^{*}=M^{*}>0$, the $\mathrm{SU}(1,1)$-orbit $S=T \sigma_{3} T^{-1}$ can be identified with the positive Hermitian matrices $M$ in $\mathrm{SU}(1,1)$. Thus, $\operatorname{Tr} S_{j} S_{j^{\prime}}=\operatorname{Tr} M_{j} M_{j^{\prime}}^{-1}$ and $\operatorname{Tr} \sigma_{3} S_{j}=\operatorname{Tr} M_{j}$, and we see that $A_{\Lambda}(S, h)>0$. Since an $\operatorname{SU}(1,1)$-symmetry emerges at $h=0$, positivity of $h$ is needed to make the integrals exist. $h$ corresponds to a magnetic field and breaks the non-compact $\mathrm{SU}(1,1)$ symmetry to $\mathrm{U}(1)$.

Our main result may be stated as follows.

Theorem 1.1. - For $d \geq 3$ space dimensions there is a constant $C_{0}$ such that

$$
\left\langle\left(\operatorname{Tr} \sigma_{3} S_{0}\right)^{2}\right\rangle_{\Lambda}(\beta, h) \leq C_{0}
$$

for all $\beta \geq 3 / 2$ and $|\Lambda| h \geq 1$.

Remark. - If the SU(1,1)-symmetry were restored in the limit $h \rightarrow 0$, the expectation of the unbounded observable $\left(\operatorname{Tr} \sigma_{3} S_{0}\right)^{2}$ would have to diverge in that limit. Our result can therefore be viewed as a statement of spontaneous symmetry breaking. (A more detailed discussion of what it means for a non-compact symmetry to be broken spontaneously has recently been given in $[\mathbf{N} \mid$.)

Higher moments of $\operatorname{Tr} \sigma_{3} S_{0}$ can also be bounded in $d \geq 3$. For $d=1,2$ we expect (but do not prove) that the same kind of bound holds except that we must require

$$
|\Lambda| \leq \begin{cases}\exp \left(C_{2} \beta\right) & d=2 \\ C_{1} \beta & d=1\end{cases}
$$

with constants $C_{1}, C_{2}$ independent of $\beta$.

Our proof can easily be extended to finite-range interactions for large $\beta$; however, for technical reasons it does not easily extend to infinite-range interactions.

In the next section we shall explain the relation between the band random matrices and the sigma models described above. Roughly speaking, the magnetic field $h$ is proportional to $\varepsilon$, the imaginary part of the energy in $(1.5)$, and $\beta$ is proportional to $W^{2}$, the square of the band width. In $d=1$, the action (1.7) just describes a random walk on $\mathrm{H}^{2}$ indexed by time $j \in \mathbb{Z}$. 
In the sigma-model approximation we shall see that

$$
\begin{aligned}
\left\langle\left\langle\left|(H-E+\mathrm{i} \varepsilon)^{-1}(x, x)\right|^{2}\right\rangle\right\rangle & \equiv \frac{\left\langle\left|(H-E+\mathrm{i} \varepsilon)^{-1}(x, x)\right|^{2}|\operatorname{Det}(H-E+\mathrm{i} \varepsilon)|^{-2}\right\rangle}{\left\langle|\operatorname{Det}(H-E+\mathrm{i} \varepsilon)|^{-2}\right\rangle} \\
& \propto\left\langle\left(\operatorname{Tr} \sigma_{3} S_{0}\right)^{2}\right\rangle_{\Lambda}(\beta, h) .
\end{aligned}
$$

Here again we identify $h$ with $\varepsilon$. The extra factors of the inverse determinant appear because we have not included the F-F and F-B sectors.

The proof of Theorem 1.1 relies on the use of horospherical coordinates $(s, t)$ to parametrize $S_{j} \in \mathrm{H}^{2}$ (for the details see Sect. 3). The action in these coordinates is

$$
A_{\Lambda}(S, h)=\sum_{i, j \in \Lambda}^{\prime}\left(\cosh \left(t_{i}-t_{j}\right)+\mathrm{e}^{t_{i}+t_{j}}\left(s_{i}-s_{j}\right)^{2}\right)+h \sum_{j \in \Lambda}\left(\cosh t_{j}+s_{j}^{2} \mathrm{e}^{t_{j}}\right) .
$$

The Gibbs measure now takes the form $\mathrm{e}^{-\beta A_{\Lambda}(S, h)} \prod_{j \in \Lambda} \mathrm{e}^{t_{j}} d t_{j} d s_{j}$.

Note that $A_{\Lambda}$ is convex in $t$ and quadratic in $s$. (We mention in passing that the BakryEmery tensor Hess + Ricci for $A_{\Lambda}$ is not positive in the natural hyperbolic geometry.) One of the key advantages of the horospherical coordinates is that we can integrate out the $s$ variables thereby producing an effective action $E_{h}(t)$. For $\beta \geq 3 / 2$ we prove that the Hessian of $E_{h}(t)$ is positive, and in fact as quadratic forms we prove

$$
E^{\prime \prime}(t)=\operatorname{Hess} E_{h}(t) \geq-\left(\beta-\frac{1}{2}\right) \Delta+h,
$$

with $\Delta$ the discrete Laplacian. Now the Brascamp-Lieb inequality may be applied to control fluctuations of the $t_{x}$ in terms of $\left(-\left(\beta-\frac{1}{2}\right) \Delta+h\right)^{-1}(x, x)$. This is bounded in three dimensions provided $|\Lambda| h \geq 1$. In one or two dimensions one must require (1.10).

The remainder of this paper is organized as follows. In Sect. 2 we describe the relation of the sigma model (1.7) to the random band model described by (1.3) following ideas of Fyodorov. Horospherical coordinates are introduced in Sect. 3 and convexity of the effective action is proved in Sect. 4 The Brascamp-Lieb inequality together with a Ward identity are used in Sect. 5 to obtain bounds on the $t$ fields. The remaining Sects. 6 and 7 explain how to control the $s$ field fluctuations and the $h$-regularization.

There are a number of open questions related to this paper. The primary one is to determine whether there are analogous bounds for more general hyperbolic sigma models such as those of higher rank. There are also problems involving averages of Green's functions which are not uniformly elliptic. Note that $s$ correlations are expressed in terms of

$$
\left\langle\left(-\nabla \mathrm{e}^{2 t} \nabla+h \mathrm{e}^{t}\right)^{-1}(x, y)\right\rangle
$$

Since the $t$ fields are not bounded from below, the Green's function (1.14) is not uniformly elliptic. The distribution of the $t$ fields is given by the convex effective action $E_{h}$. Although we obtain good bounds on (1.14) for the diagonal $x=y$, the off-diagonal bounds obtained by our methods are not sharp. 
Acknowledgments: T. Spencer would like to thank M. Disertori, K. Gawedzki, G. Papanicolau and S.R.S. Varadhan for helpful comments.

\section{Origin of the model}

We now review how the non-linear sigma model, (1.7) and (1.8), arises from the problem of computing Green's function averages for some ensembles of band random matrices. Aside from putting our analysis on a solid footing in random-matrix theory and disordered electron physics, this review will explain the origin of the hyperbolic target space $\mathrm{H}^{2}$ and its Riemannian geometry.

Readers interested only in mathematical results, not in physical motivation and background, are invited to skip the present section; the remainder of the paper does not depend on it.

2.1. Gaussian ensembles of band random matrices. - Let $\Lambda \subset \mathbb{Z}^{d}$ be a periodic box as before, and assign to every site $i \in \Lambda$ one copy $V_{i}$ of an $N$-dimensional unitary vector space. (Physically speaking we are assigning $N$ valence electron orbitals to every atom of a solid with hypercubic lattice structure.) The finite-dimensional Hilbert space $V$ of the random-matrix model to be specified is the orthogonal sum

$$
V=V_{1} \oplus V_{2} \oplus \ldots \oplus V_{|\Lambda|} .
$$

The basic framework we have in mind is single-electron quantum mechanics, and our goal is to establish control over the spectral and transport properties of certain ensembles of random Hamiltonians $H$. We shall take the Hamiltonians to be elements of $\operatorname{Herm}(V)$, the space of Hermitian operators on $V$.

A random-matrix model is now defined by a probability distribution on $\operatorname{Herm}(V)$. Equivalently, one may specify the Fourier transform or characteristic function:

$$
\Omega(K)=\left\langle\mathrm{e}^{\mathrm{i} \operatorname{Tr} H K}\right\rangle,
$$

where $\langle\ldots\rangle$ denotes the expectation value w.r.t. the probability distribution for the random Hamiltonian $H$. The Fourier variable is some other element $K \in \operatorname{Herm}(V)$.

For simplicity we shall restrict ourselves to the case of Gaussian distributions with zero mean, $\langle H\rangle=0$. If $\Pi_{i}$ is the orthogonal projector on the linear subspace $V_{i} \subset V$, we take the characteristic function to be

$$
\Omega(K)=\exp \left(-\frac{1}{2} \sum_{i, j=1}^{|\Lambda|} J_{i j} \operatorname{Tr}\left(\Pi_{i} K \Pi_{j} K\right)\right),
$$

where the coefficients $J_{i j}$ are real, symmetric, and non-negative (they must also be positive semi-definite as a quadratic form). The choice (2.2) also implies that all matrix entries of $H$ are statistically independent.

We mention in passing that the characteristic function (2.2) is invariant under conjugation $K \mapsto U K U^{-1}$ by $U \in \mathcal{U}$ where $\mathcal{U}$ is the direct product of all the groups of 
unitary transformations in the subspaces:

$$
\mathcal{U}=\mathrm{U}\left(V_{1}\right) \times \mathrm{U}\left(V_{2}\right) \times \cdots \times \mathrm{U}\left(V_{|\Lambda|}\right) .
$$

This means that the probability distribution for the Hamiltonian $H$ has a local gauge invariance. Models of this kind were first introduced and studied by Wegner [W1].

By varying the lattice $\Lambda$, the number of orbitals $N$, and the variances $J_{i j}$, one obtains a large class of Hermitian random-matrix models. In particular, if $d(i, j)=|i-j|$ denotes a distance function for $\Lambda$, and $f$ is a rapidly decreasing positive function on $\mathbb{R}_{+}$of width $W$, the choice $J_{i j}=f(|i-j|)$ gives an ensemble of band random matrices with band width $W$ and symmetry group $\mathcal{U}=\mathrm{U}(N)^{|\Lambda|}$.

There exist two distinct situations where such a random-matrix ensemble is expected to exhibit metallic behavior (in dimension $d \geq 3$ ) and efficient methods of analysis are available. The first one occurs when the width $W$ is large (and, say, $N=1$ ). This case is dealt with by the Schäfer-Wegner method $[\mathbf{S}]$; it will not be considered further in the present paper (see however $[\mathbf{Z}]$ for a recent review of that method).

The second one is called the 'granular model'. There, $N \gg 1$ and the diagonal of the variance matrix $J_{i j}$ dominates the off-diagonal:

$$
J_{i i} \gg \sum_{j: j \neq i} J_{i j}
$$

Each atom $i \in \Lambda$ here is to be viewed as a grain, or small metallic particle, housing a large number $N$ of electron states, and the squared matrix elements for tunneling between grains $\left(J_{i j}\right.$ for $\left.i \neq j\right)$ are small compared to the intra-grain matrix elements $\left(J_{i i}\right)$. The appropriate method to use in this case is that of Fyodorov (Sect. 2.3). Metallic behavior is expected to occur when the coefficients $N J_{i j} / \sqrt{J_{i i} J_{j j}}$ are not too small.

Another (perhaps more familiar) way of defining the class of granular models is to say that one starts from matrices $H$ drawn from the Gaussian Unitary Ensemble (GUE) of matrix dimension $N|\Lambda|$, partitions $V=\mathbb{C}^{N|\Lambda|}$ as $V=V_{1} \oplus V_{2} \oplus \ldots \oplus V_{|\Lambda|}$, and then multiplies the variances of all matrix elements of $H$ connecting $V_{i} \simeq \mathbb{C}^{N}$ with $V_{j} \simeq \mathbb{C}^{N}$ by the positive number $J_{i j}$. This is the same as the model $(1.3)$ described in the introduction, with $N$ being equal to the volume of the cubes tiling the lattice.

2.2. Averaging the Green's functions over the disorder. - Fixing some lattice site $\ell \in \Lambda$, let $A_{\ell}$ be the average absolute square of the $(\ell, \ell)$ part of the Green's function with complex energy $E-$ i $\varepsilon$ :

$$
A_{\ell}(E, \varepsilon)=\left\langle\left|\operatorname{Tr}(H-E+\mathrm{i} \varepsilon)^{-1} \Pi_{\ell}\right|^{2}\right\rangle .
$$

Physicists have developed an approximation scheme that calculates disorder averages such as this one by relating them to the correlation functions of a supersymmetric nonlinear sigma model [E1]. Here we shall address the related, but somewhat simpler 
problem that arises from considering

$$
B_{\ell}^{(n)}(E, \varepsilon)=\frac{\left\langle\left|\operatorname{Tr}(H-E+\mathrm{i} \varepsilon)^{-1} \Pi_{\ell}\right|^{2} \times|\operatorname{Det}(H-E+\mathrm{i} \varepsilon)|^{-2 n}\right\rangle}{\left\langle|\operatorname{Det}(H-E+\mathrm{i} \varepsilon)|^{-2 n}\right\rangle} .
$$

Note that $B_{\ell}^{(n)}$ can be viewed as the average squared Green's function for a deformed ensemble, where the probability distribution for the Hamiltonian $H$ is modified by multiplying it with $2 n$ inverse powers of $|\operatorname{Det}(H-E+\mathrm{i} \varepsilon)|$. Inserting these factors modifies the original problem, and it is far from clear how much bearing the results for $n \geq 1$ will have on the case $n=0$. (Physicists often use a procedure called the replica trick, where one attempts to infer the answer for $n=0$ by analytic continuation from the answer for $n \in \mathbb{N}$. We will make no such attempt here.) However, even if the $n \geq 1$ situation was quite unrelated to $n=0$, analysing it would still be a necessary step toward establishing mathematical control over the full supersymmetric theory at $n=0$. The reason is that Efetov's supersymmetric non-linear sigma model has the effective theory of $B_{\ell}^{(n)}$ at $n=1$ for its non-compact bosonic sector.

In order to express $B_{\ell}^{(1)}$ we introduce a pair of complex fields $\phi=\left(\phi_{+}, \phi_{-}\right)$where $\phi_{ \pm} \in V$. The projections $\Pi_{j} \phi_{+}=\phi_{+}(j)$ and $\Pi_{j} \phi_{-}=\phi_{-}(j)$ are complex $N$-component vectors. The scalar product is given by

$$
\left(\phi_{+}^{*}, \phi_{+}\right)=\sum_{j} \phi_{+}^{*}(j) \cdot \phi_{+}(j) .
$$

If $A$ is a matrix or linear operator acting on $V$ with $\mathfrak{R e} A=\frac{1}{2}\left(A+A^{*}\right)>0$, we normalize our Gaussian integrals over $\phi$ such that

$$
\int \mathrm{e}^{-\left(\phi_{+}^{*}, A \phi_{+}\right)}=\operatorname{Det}^{-1}(A) .
$$

For fixed $E, \varepsilon$ and $\Lambda$ define the quadratic form

$$
\left(\phi^{*}, A_{H} \phi\right)=-\mathrm{i}\left(\phi_{+}^{*},(H-E+\mathrm{i} \varepsilon) \phi_{+}\right)+\mathrm{i}\left(\phi_{-}^{*},(H-E-\mathrm{i} \varepsilon) \phi_{-}\right) .
$$

Note that $\mathfrak{R e} A \geq \varepsilon$ and the integral over $\phi$ is therefore defined:

$$
Z_{\Lambda} \equiv \int \mathrm{e}^{-\left(\phi^{*}, A_{H} \phi\right)}=|\operatorname{Det}(H-E+\mathrm{i} \varepsilon)|^{-2}
$$

and

$$
\int \mathrm{e}^{-\left(\phi^{*}, A_{H} \phi\right)}\left|\phi_{+}(\ell)\right|^{2}\left|\phi_{-}(\ell)\right|^{2}=\left|\operatorname{Tr}(H-E+\mathrm{i} \varepsilon)^{-1} \Pi_{\ell}\right|^{2}|\operatorname{Det}(H-E+\mathrm{i} \varepsilon)|^{-2} .
$$

For general $n \geq 1$ if we set $\phi_{ \pm}(j)=\left\{\phi_{ \pm 1}(j), \phi_{ \pm 2}(j), \ldots, \phi_{ \pm n}(j)\right\}$, this produces the factor $|\operatorname{Det}(H-E+\mathrm{i} \varepsilon)|^{-2 n}$ and permits us to study more complicated observables involving several Green's functions.

Now we can calculate the average of $Z_{\Lambda}$ over the randomness in $H$ by using (2.2). First consider

$$
\left\langle\mathrm{e}^{\mathrm{i}\left(\phi_{+}^{*}, H \phi_{+}\right)-\mathrm{i}\left(\phi_{-}^{*}, H \phi_{-}\right)}\right\rangle=\mathrm{e}^{-\frac{1}{2} \sum J_{i j} \operatorname{Tr}\left(s M_{i} s M_{j}\right)}
$$


where

$$
M_{j}=\left(\begin{array}{ll}
\phi_{+}^{*}(j) \cdot \phi_{+}(j) & \phi_{+}^{*}(j) \cdot \phi_{-}(j) \\
\phi_{-}^{*}(j) \cdot \phi_{+}(j) & \phi_{-}^{*}(j) \cdot \phi_{-}(j)
\end{array}\right),
$$

and $s=\sigma_{3}=\operatorname{diag}(1,-1)$. For general $n, \sigma_{3}$ is replaced by the diagonal matrix $s=$ $\operatorname{diag}\left(\operatorname{Id}_{n},-\operatorname{Id}_{n}\right)$. Note that the $2 n \times 2 n$ matrices $M_{j}$ are Hermitian and non-negative; we say they lie in $\operatorname{Herm}^{+}\left(\mathbb{C}^{2 n}\right)$.

2.3. Fyodorov's method. - Following Fyodorov $[\mathbf{F}]$ we choose the matrices $M_{j}$ as our integration variables, i.e., we push forward the measure over the $\phi$ to a measure over the non-negative matrices $M$. The push forward may be singular. However, if $N \geq 2 n$ then the push forward has a density (derived in Appendix A) given by

$$
\prod_{i=1}^{|\Lambda|} \operatorname{Det}^{N-2 n}\left(M_{i}\right) d M_{i}
$$

where $d M_{i}$ denotes a (suitably normalized) Lebesgue measure on $\operatorname{Herm}^{+}\left(\mathbb{C}^{2 n}\right)$. Now set

$$
d \mu\left(M_{i}\right):=\operatorname{Det}^{-2 n}\left(M_{i}\right) d M_{i}
$$

Then we obtain Fyodorov's formula for $Z_{\Lambda}$ (for general $n$ ) in the form

$$
Z_{\Lambda}=\int \mathrm{e}^{-\frac{1}{2} \sum_{i j} J_{i j} \operatorname{Tr}\left(s M_{i} s M_{j}\right)} \prod_{k \in \Lambda} \mathrm{e}^{\operatorname{Tr}(\mathrm{i} s E-\varepsilon) M_{k}} \operatorname{Det}^{N}\left(M_{k}\right) d \mu\left(M_{k}\right),
$$

where the integral is over the configurations $\left\{M_{i}\right\}$ with $M_{i}>0$ for all $i \in \Lambda$.

The formulation (2.9) offers a transparent view of the symmetries of the problem. Indeed, let $\mathrm{U}(n, n)$ be the pseudo-unitary group of complex $2 n \times 2 n$ matrices $T$ with inverse $T^{-1}=s T^{*}$. This group acts as a transformation group on the matrices $M_{i}$ by

$$
M_{i} \mapsto T M_{i} T^{*}
$$

Clearly, the integration domain for $M_{i} \in \operatorname{Herm}^{+}\left(\mathbb{C}^{2 n}\right)$ of Fyodorov's formula (2.9) is invariant under that group action. Since $|\operatorname{Det} T|=1$ for $T \in \mathrm{U}(n, n)$, the same holds true for the integration density $d \mu\left(M_{i}\right)$. From

$$
M_{i} s \mapsto T M_{i} T^{*} s=T M_{i} s T^{-1}
$$

one sees that the function being integrated in (2.9) becomes invariant under the $\mathrm{U}(n, n)$ group action when the parameter $\varepsilon$ is taken to zero. Thus $\mathrm{U}(n, n)$ transformations are global symmetries in that limit.

In what follows we focus on the case $n=1$, where the symmetry group is $\mathrm{U}(1,1)$ or, what amounts to essentially the same, $\mathrm{SU}(1,1)$. 
2.4. The sigma-model approximation. - The exact integral representation (2.9) is well suited for further analysis in the granular limit which we now consider. Thus we now assume $N \gg 1, J_{i j}=0$ for $|i-j| \geq 2, J_{i j}=J_{1}>0$ for $|i-j|=1$, and

$$
J_{i i} \equiv J_{0} \gg 2 J_{1} d \text {. }
$$

Let us first consider the diagonal terms of the action (2.9):

$$
\sum_{j \in \Lambda}\left(\frac{1}{2} J_{0} \operatorname{Tr}\left(s M_{j}\right)^{2}-\mathrm{i} E \operatorname{Tr}\left(s M_{j}\right)-N \operatorname{Tr} \ln M_{j}\right) .
$$

The matrices $M_{j} s$ may be expressed as

$$
M_{j} s=T_{j}\left(\begin{array}{cc}
p_{1}(j) & 0 \\
0 & -p_{2}(j)
\end{array}\right) T_{j}^{-1},
$$

where $p_{1}(j), p_{2}(j)$ are positive real numbers, and $T_{j} \in \mathrm{SU}(1,1)$ is determined only up to right multiplication by an arbitrary element in $K \equiv \mathrm{U}(1)$. The measure becomes

$$
d \mu\left(M_{j}\right)=\frac{\left(p_{1}(j)+p_{2}(j)\right)^{2}}{p_{1}(j)^{2} p_{2}(j)^{2}} d p_{1}(j) d p_{2}(j) d \mu_{K}\left(T_{j}\right)
$$

where $d \mu_{K}\left(T_{j}\right)$ is an invariant measure for $\mathrm{SU}(1,1) / \mathrm{U}(1)$. The diagonal terms of the action can be written in terms of $p_{1}, p_{2}$ :

$$
\frac{J_{0}}{2}\left(p_{1}^{2}+p_{2}^{2}\right)-\mathrm{i} E\left(p_{1}-p_{2}\right)-N\left(\ln p_{1}+\ln p_{2}\right) \text {. }
$$

The critical point for $E^{2} \leq 4 N J_{0}$ is given by

$$
p_{1}=\frac{\mathrm{i} E}{2 J_{0}}+\rho_{N}(E), \quad \rho_{N}(E)=\frac{\sqrt{4 N J_{0}-E^{2}}}{2 J_{0}},
$$

and $p_{2}=\bar{p}_{1}$, where $\rho_{N}$ is the mean-field expression for the local density of states (but for a factor of $\pi$ ). In this approximation,

$$
M_{j} s=T_{j}\left(\begin{array}{cc}
\rho_{N}(E) & 0 \\
0 & -\rho_{N}(E)
\end{array}\right) T_{j}^{-1}+\text { const } \times \text { Id } .
$$

The last term arising from the imaginary part of $p_{1}, p_{2}$ is of no consequence. Thus

$$
M_{j} s=\rho_{N}(E) S_{j},
$$

where $S_{j}$ was defined by $S_{j}=T_{j} \sigma_{3} T_{j}^{-1}$ as before. The action function of our model now is

$$
A_{\Lambda}(S)=\frac{1}{2} \beta \sum_{\Lambda}^{\prime} \operatorname{Tr}\left(S_{j} S_{j^{\prime}}\right)+\frac{1}{2} h \sum_{j \in \Lambda} \operatorname{Tr}\left(\sigma_{3} S_{j}\right),
$$

with $\beta=2 J_{1} \rho_{N}^{2}(E)$ and $h=2 \varepsilon \rho_{N}(E)$. Similarly, by using (2.7) and (2.14), the observable appearing in (2.5) is proportional to $\left(\operatorname{Tr} \sigma_{3} S_{0}\right)^{2}$ in the sigma-model approximation. The $\mathrm{SU}(1,1)$-invariant measure $d \mu_{K}\left(T_{j}\right)$ is renamed to $d \mu\left(S_{j}\right)$.

In order to eliminate the sigma-model approximation we must control the massive fluctuations of $p_{1}(j), p_{2}(j)$ about the saddle. Although the Gibbs measure is complex, 
if we integrate over these eigenvalues a new effective action is produced which is real. This new effective action may share the desired convexity properties with $A_{\Lambda}(S)$.

\section{The model in horospherical coordinates}

Having clarified the origin of the hyperbolic non-linear sigma model in disordered electron physics, we now begin our study of it. In the present section we introduce a coordinate system that takes advantage of the hyperbolic structure of $\mathrm{H}^{2}$ and is well suited for the purpose of doing analysis on the sigma model, Eqs. (1.7) and (1.8).

For any connected and simply connected noncompact Lie group $G$ with semisimple Lie algebra there exists an Iwasawa decomposition $[\mathbf{H}]$

$$
G=N A K,
$$

where $K, A$, and $N$ are maximal compact, maximal Abelian and nilpotent subgroups, respectively. In the case at hand, namely $G=\mathrm{SU}(1,1)$ with Lie algebra

$$
\mathfrak{s u}(1,1)=\left\{x_{1} \sigma_{1}+x_{2} \sigma_{2}+\mathrm{i} x_{3} \sigma_{3} \mid\left(x_{1}, x_{2}, x_{3}\right) \in \mathbb{R}^{3}\right\},
$$

$K$ is the $\mathrm{U}(1)$ subgroup generated by $\mathrm{i} \sigma_{3}$, and $\sigma_{i}$ are the Pauli matrices. We choose $A \simeq \mathbb{R}^{+}$to be the Abelian group generated by $\sigma_{1}$; the nilpotent group $N$ then is the one-parameter group with nilpotent generator $\sigma_{2}-\mathrm{i} \sigma_{3}$. Passing to equivalence classes or cosets by the right action of $K=\mathrm{U}(1)$ on both sides of the Iwasawa decomposition, one gets an identification

$$
\mathrm{H}^{2} \simeq \mathrm{SU}(1,1) / \mathrm{U}(1) \simeq N A \cdot o
$$

Thus the two-hyperboloid $\mathrm{H}^{2}$ is viewed as the orbit of the one-parameter groups $N$ and $A$ acting on the coset $o=K$.

Introducing two real variables $s$ and $t$, we parameterize the Lie groups $N$ and $A$ as

$$
N=\left\{n_{s}=\mathrm{e}^{s\left(\sigma_{2}-\mathrm{i} \sigma_{3}\right) / 2} \mid s \in \mathbb{R}\right\}, \quad A=\left\{a_{t}=\mathrm{e}^{t \sigma_{1} / 2} \mid t \in \mathbb{R}\right\} .
$$

We refer to $s$ and $t$ as horospherical coordinates. Their relation to the matrix $S$ parameterizing $\mathrm{H}^{2}$ is given by

$$
S \sigma_{3}=n_{s} a_{t}\left(n_{s} a_{t}\right)^{*}=\left(\begin{array}{cc}
\cosh t+\frac{s^{2}}{2} \mathrm{e}^{t} & \sinh t-\left(\text { is }+\frac{s^{2}}{2}\right) \mathrm{e}^{t} \\
\sinh t+\left(\text { is }-\frac{s^{2}}{2}\right) \mathrm{e}^{t} & \cosh t+\frac{s^{2}}{2} \mathrm{e}^{t}
\end{array}\right)
$$

and the $\mathrm{SU}(1,1)$-invariant metric tensor $g$ in these coordinates takes the form

$$
g=\frac{1}{2} \operatorname{Tr} \mathrm{d} S^{2}=\mathrm{d} t^{2}+\mathrm{e}^{2 t} \mathrm{~d} s^{2} .
$$

How does the action of the subgroups $N, A$, and $K$ on $\mathrm{H}^{2}$ look in horospherical coordinates? (These group actions are important because they furnish global symmetries of the non-linear sigma model in the limit of vanishing regularization, $h \rightarrow 0$.) First of all, since $N$ is a one-parameter group one has

$$
n_{s_{0}}\left(n_{s} a_{t}\right) \cdot o=n_{s+s_{0}} a_{t} \cdot o
$$


so $n_{s_{0}} \in N$ acts on $n_{s} a_{t} \cdot o \in N A \cdot o$ by simply translating $(s, t) \mapsto\left(s+s_{0}, t\right)$. Second, from the fact that $\sigma_{2}-\mathrm{i} \sigma_{3}$ is an eigenvector of the commutator action $\left[\sigma_{1}, \cdot\right]$ with eigenvalue -2 , one easily verifies

$$
a_{t_{0}}\left(n_{s} a_{t}\right) \cdot o=\left(a_{t_{0}} n_{s} a_{-t_{0}}\right)\left(a_{t_{0}} a_{t}\right) \cdot o=n_{\mathrm{e}^{-t_{0} s}} a_{t+t_{0}} \cdot o,
$$

so $a_{t_{0}} \in A$ acts by $(s, t) \mapsto\left(\mathrm{e}^{-t_{0}} s, t+t_{0}\right)$. Third, the group action of $K$ in horospherical coordinates is somewhat complicated and will not be considered here.

The energy or action function of the non-linear sigma model (1.8) in horospherical coordinates is expressed by

$$
A_{\Lambda}=\beta \sum_{\Lambda}^{\prime}\left(\cosh \left(t_{i}-t_{i^{\prime}}\right)+\frac{1}{2}\left(s_{i}-s_{i^{\prime}}\right)^{2} \mathrm{e}^{t_{i}+t_{i^{\prime}}}\right)+h \sum_{j \in \Lambda}\left(\cosh t_{j}+\frac{1}{2} s_{j}^{2} \mathrm{e}^{t_{j}}\right) .
$$

The Gibbs measure is

$$
d \mu_{\Lambda, A}=\mathrm{e}^{-A_{\Lambda}} \prod_{i \in \Lambda} \mathrm{e}^{t_{i}} d t_{i} d s_{i}
$$

As expected, $d \mu_{\Lambda, A}$ becomes invariant under global $N$ and $A$ transformations,

$$
\begin{array}{rlll}
n_{s}: & \left(s_{i}, t_{i}\right) & \mapsto & \left(s_{i}+s, t_{i}\right), \\
a_{t}: & \left(s_{i}, t_{i}\right) & \mapsto & \left(\mathrm{e}^{-t} s_{i}, t_{i}+t\right),
\end{array}
$$

in the limit $h \rightarrow 0$.

Our observable given in Theorem 1.1 may be expressed as

$$
\left(\operatorname{Tr} \sigma_{3} S_{0}\right)^{2}=\left(2 \cosh t_{0}+s_{0}^{2} \mathrm{e}^{t_{0}}\right)^{2} .
$$

Note that since the action is quadratic in $s$, the integral over the variable $s_{0}$ is Gaussian and can be done explicitly.

\section{Integration of the $s$ fields}

In this section we shall analyse the action (3.3). Since it is quadratic in the $s$ fields, they can be integrated out. Consider the interaction between the $s$ and $t$ fields in (3.3) and define

$$
B(s, t)=\sum_{\Lambda}^{\prime} \mathrm{e}^{t_{i}+t_{j}}\left(s_{i}-s_{j}\right)^{2} \equiv(s, D s)_{\Lambda},
$$

where $(f, g)_{\Lambda} \equiv \sum_{i \in \Lambda} f_{i} g_{i}$, and $D$ is a matrix corresponding to an elliptic operator with periodic boundary conditions and with coefficients that depend on $t_{i}$. As a quadratic form $D$ is non-negative, and its matrix elements are given by

$$
D_{i j}=\left\{\begin{array}{ll}
-\mathrm{e}^{t_{i}+t_{j}} & |i-j|=1 \\
0 & |i-j|>1
\end{array} \quad, \quad D_{i i}=-\sum_{j: j \neq i} D_{i j} .\right.
$$

When the variables $t_{i}$ all vanish, $D=-\Delta_{\Lambda}$ where $\Delta_{\Lambda}$ is the discrete Laplacian of the lattice $\Lambda$ with periodic boundary conditions. Although $D$ is elliptic, it is not uniformly elliptic as the $\left|t_{i}\right|$ may be very large. 
Using (4.1) and integrating over the $s$ fields we obtain an explicit expression for the effective action:

$$
E_{h}=\beta \sum_{\Lambda}^{\prime} \cosh \left(t_{i}-t_{i^{\prime}}\right)+C_{h}(t)+\sum_{j \in \Lambda}\left(-t_{j}+h \cosh t_{j}\right)
$$

where

$$
C_{h}(t)=\frac{1}{2} \ln \operatorname{Det}\left(D(t)+h \mathrm{e}^{t}\right)+\mathrm{const}=-\ln \int \mathrm{e}^{-\frac{\beta}{2} B(s, t)-\frac{h}{2} \sum \mathrm{e}^{t} s^{2}} \prod_{i \in \Lambda} d s_{i} .
$$

We are going to regard the torus variables $t_{i}$ as Cartesian coordinates of $\mathbb{R}^{|\Lambda|}$ equipped with the canonical Euclidean geometry, and have therefore relocated the variable volume factors $\mathrm{e}^{t_{i}}$ from $\prod \mathrm{e}^{t_{i}} d t_{i}$ to $E_{h}$. Notice that the effective Gibbs measure

$$
\mathrm{e}^{-E_{h}} \prod_{i \in \Lambda} d t_{i}
$$

for $h=0$ is invariant under shifts $t_{i} \rightarrow t_{i}+\gamma$. This invariance is a remnant of the global symmetry (3.4) of the original theory, and will play an important role in later discussions.

We shall first analyse a slightly different expression

$$
\mathrm{e}^{-\widetilde{C}(t)}=\int \mathrm{e}^{-\frac{\beta}{2} B(s, t)} \delta\left(\sum_{i \in \Lambda} s_{i}\right) \prod_{j \in \Lambda} d s_{j},
$$

where the $\delta$-function eliminates the zero mode of $B$ and makes the integral exist. By Gaussian integration we have

$$
\widetilde{C}(t)=\frac{1}{2} \ln \operatorname{Det} \widetilde{D}(t),
$$

where $\widetilde{D}>0$ is $D$ acting on the orthogonal complement of the constant functions. Both $\widetilde{C}$ and $\widetilde{D}$ depend on $\Lambda$ and the $t$ field, but we shall frequently omit these dependences for notational brevity. The effective action in $t$ is

$$
E_{\Lambda}=\beta \sum_{\Lambda}^{\prime} \cosh \left(t_{i}-t_{i^{\prime}}\right)+\widetilde{C}(t)+\sum_{j \in \Lambda}\left(-t_{j}+h \cosh t_{j}\right)
$$

We shall set the factor of $\beta / 2$ appearing in (4.5) equal to 1 . By scaling in $s$ this simply shifts $\widetilde{C}(t)$ by a trivial constant.

For a function $F$ of $t_{i}(i \in \Lambda)$ let the Euclidean Hessian of $F$ be denoted by $F^{\prime \prime}$ :

$$
F_{i j}^{\prime \prime}=\frac{\partial^{2} F}{\partial t_{i} \partial t_{j}} \quad(i, j \in \Lambda) .
$$

Theorem 4.1. - For any value of the coupling parameter $\beta \geq 3 / 2$ and dimension $d \geq 1$ the function $E_{\Lambda}$ is convex and

$$
E_{\Lambda}^{\prime \prime} \geq-\left(\beta-\frac{1}{2}\right) \Delta_{\Lambda}+h \geq-\Delta_{\Lambda}+h .
$$

Proof. - Clearly from (4.6)

$$
E_{\Lambda}^{\prime \prime} \geq-\beta \Delta_{\Lambda}+h+\widetilde{C}^{\prime \prime}
$$


so it suffices to estimate $\widetilde{C}^{\prime \prime}$. From 4.5 with $\beta / 2$ set to 1 we have

$$
\frac{\partial \widetilde{C}}{\partial t_{i}}=\left\langle U_{i}\right\rangle_{s}, \quad U_{i}=\sum_{j:|i-j|=1} \mathrm{e}^{t_{i}+t_{j}}\left(s_{i}-s_{j}\right)^{2},
$$

where $\langle\cdot\rangle_{s}$ denotes the average over the $s$ field with Gibbs weight $\mathrm{e}^{-B} \delta$. For $|i-j|>1$ we have

$$
-\frac{\partial^{2} \widetilde{C}}{\partial t_{i} \partial t_{j}}=\left\langle U_{i} ; U_{j}\right\rangle_{s} \equiv\left\langle U_{i} U_{j}\right\rangle_{s}-\left\langle U_{i}\right\rangle_{s}\left\langle U_{j}\right\rangle_{s},
$$

and for $|i-j|=1$

$$
-\frac{\partial^{2} \widetilde{C}}{\partial t_{i} \partial t_{j}}=\left\langle U_{i} ; U_{j}\right\rangle_{s}-\left\langle\mathrm{e}^{t_{i}+t_{j}}\left(s_{i}-s_{j}\right)^{2}\right\rangle_{s},
$$

while on the diagonal

$$
-\frac{\partial^{2} \widetilde{C}}{\partial t_{i}^{2}}=\left\langle U_{i} ; U_{i}\right\rangle_{s}-\left\langle U_{i}\right\rangle_{s}
$$

Let

$$
K_{i j}=\left\langle U_{i} ; U_{j}\right\rangle_{s},
$$

and decompose $\widetilde{C}^{\prime \prime}$ into two pieces:

$$
\widetilde{C}_{i j}^{\prime \prime}=\left(2\left\langle U_{i}\right\rangle_{s} \delta_{i j}-K_{i j}\right)+R_{i j}
$$

where $R$ is a local remainder term.

By explicit computation $K_{i j}(t) \geq 0$ for all $i, j$ and field configurations $t$, because the square of a Green's function arises.

Lemma 4.2. - With $U$ and $K$ defined by (4.8) and (4.9) we have for every $t$

$$
\sum_{j} K_{i j}=2\left\langle U_{i}\right\rangle_{s}
$$

This relation implies that as a quadratic form

$$
2\left\langle U_{i}\right\rangle_{s} \delta_{i j}-K_{i j} \geq 0
$$

Proof. - To get (4.11) make the change of variables $s_{k} \rightarrow s_{k} \mathrm{e}^{\gamma}(k \in \Lambda)$. Then $U_{i} \rightarrow$ $U_{i} \mathrm{e}^{2 \gamma}$ and $B \rightarrow B \mathrm{e}^{2 \gamma}$, while the expectation value $\left\langle U_{i}\right\rangle_{s}$ remains invariant. Differentiating $\left\langle U_{i}\right\rangle_{s}$ with respect to $\gamma$ at $\gamma=0$ yields

$$
0=2\left\langle U_{i}\right\rangle_{s}-2\left\langle U_{i} ; B\right\rangle,
$$

and since $B=\frac{1}{2} \sum_{j} U_{j}$ we obtain 4.11).

The non-negativity of the quadratic form (4.12) now follows from the Schwarz inequality:

$$
\left|\sum_{i, j} K_{i j} f_{i} f_{j}\right| \leq\left[\sum_{i, j} K_{i j} f_{j}^{2}\right]^{\frac{1}{2}}\left[\sum_{i, j} K_{i j} f_{i}^{2}\right]^{\frac{1}{2}}=2 \sum_{i} U_{i} f_{i}^{2} .
$$


Here we used the pointwise positivity of $K_{i j}$ to write the first expression as a scalar product of two vectors $u$ and $v$ with components $u_{i j}=\sqrt{K_{i j}} f_{j}$ and $v_{i j}=\sqrt{K_{i j}} f_{i}$.

We now must estimate the remaining local part $R=\widetilde{C}^{\prime \prime}-2\langle U\rangle \delta+K$, which is expressed by

$$
R_{i j}=-\left\langle U_{i}\right\rangle_{s} \delta_{i j}+\mathrm{e}^{t_{i}+t_{j}}\left\langle\left(s_{i}-s_{j}\right)^{2}\right\rangle_{s} \delta(|i-j|-1) .
$$

Note that $R \leq 0$ as a quadratic form and that $\sum_{j} R_{i j}=0$ for each $i$.

Lemma 4.3. - For all real $f_{i}$

$$
\left|\sum_{i, j} R_{i j} f_{i} f_{j}\right| \leq \frac{1}{2} \sum_{i}(\nabla f)_{i}^{2},
$$

where $\nabla f$ denotes the discrete gradient of the lattice $\Lambda$.

Proof. - The left-hand side of (4.13) can be written as a sum over nearest-neighbor pairs $i, j$ :

$$
\sum_{|i-j|=1}^{\prime} R_{i j}\left(f_{i}-f_{j}\right)^{2}
$$

It therefore suffices to show that for each pair $i, j$ we have

$$
\mathrm{e}^{t_{i}+t_{j}}\left\langle\left(s_{i}-s_{j}\right)^{2}\right\rangle_{s} \leq 1 / 2
$$

This result follows from the fact that $\langle\cdot\rangle_{s}$ is a Gaussian expectation in $s$ with terms $\mathrm{e}^{t_{i}+t_{j}}\left(s_{i}-s_{j}\right)^{2}$ appearing in the action, $B$. Indeed, if $u, v_{1}, v_{2}, \ldots$ are real variables it is a general fact that

$$
\frac{\int c u^{2} \mathrm{e}^{-c u^{2}-Q(u, v)} d u \prod_{a} d v_{a}}{\int \mathrm{e}^{-c u^{2}-Q(u, v)} d u \prod_{a} d v_{a}} \leq 1 / 2
$$

for any positive constant $c$ and any $Q \geq 0$ which is quadratic in $u, v$. If we set $c u^{2}=$ $\left(s_{i}-s_{j}\right)^{2} \mathrm{e}^{t_{i}+t_{j}}$ and $Q=B-c u^{2}$ (restricted to the linear subspace given by the constraint $\left.\sum_{i \in \Lambda} s_{i}=0\right)$, we obtain the desired result.

From the decomposition (4.10) and the two lemmas we have

$$
\widetilde{C}^{\prime \prime} \geq R \geq \frac{1}{2} \Delta_{\Lambda}
$$

Inserting this bound into (4.7) completes the proof of Theorem 4.1

\section{The Brascamp-Lieb Inequality}

We now state the Brascamp-Lieb inequality $[\mathbf{B}]$ in a form in which we shall apply it. Let $A=A(t)$ be a convex function of $N$ variables $t=\left(t_{1}, \ldots, t_{N}\right) \in \mathbb{R}^{N}$, where $\mathbb{R}^{N}$ is the Euclidean vector space with scalar product

$$
(\varphi, t)=\sum_{i=1}^{N} \varphi_{i} t_{i}
$$


With the function $A$ associate the measure $d \mu_{A}(t)=\mathrm{e}^{-A(t)} \prod d t_{i}$. Assume that the Euclidean Hessian of $A$ satisfies

$$
A^{\prime \prime}(t) \geq H>0,
$$

where $H$ is a positive $N \times N$ matrix independent of $t$.

Theorem 5.1 (Brascamp-Lieb). — If A satisfies (5.1) then

$$
\left\langle\mathrm{e}^{(\varphi, t)}\right\rangle_{A}=\frac{\int \mathrm{e}^{(\varphi, t)} \mathrm{e}^{-A(t)} \Pi^{N} d t_{i}}{\int \mathrm{e}^{-A(t)} \Pi^{N} d t_{i}} \leq \mathrm{e}^{\langle(\varphi, t)\rangle_{A}} \mathrm{e}^{\frac{1}{2}\left(\varphi, H^{-1} \varphi\right)} .
$$

For our application we identify $A$ with the function $E=E_{\Lambda}(t)$ given by (4.6). Theorem 4.1 tells us to put $H=-\left(\beta-\frac{1}{2}\right) \Delta_{\Lambda}+h$. If we then set

$$
G_{i j}=\left(-\left(\beta-\frac{1}{2}\right) \Delta_{\Lambda}+h\right)^{-1}
$$

and fix a site $i \in \Lambda$ with field variable $t_{i}$, we have

$$
\left\langle\mathrm{e}^{\alpha t_{i}-\alpha\left\langle t_{i}\right\rangle_{E}}\right\rangle_{E} \leq \mathrm{e}^{\frac{1}{2} \alpha^{2} G_{i i}}
$$

In dimension $d \geq 3$ and for $\beta \geq 3 / 2$ the Green's function $G_{i i}$ is uniformly bounded as $\Lambda \rightarrow \mathbb{Z}^{d}$ provided $h|\Lambda| \geq 1$.

Now we drop the subscript $E$ and let $\langle\cdot\rangle=\langle\cdot\rangle_{E}$. Large fluctuations of the field $t$ away from its average are very unlikely:

$$
p=\operatorname{Prob}_{E}\left\{t_{i}-\left\langle t_{i}\right\rangle \geq \rho\right\} \leq \mathrm{e}^{-\frac{\rho^{2}}{2 G_{i i}}} .
$$

Indeed, when $t_{i}-\left\langle t_{i}\right\rangle \geq \rho$ we have $\mathrm{e}^{\alpha\left(t_{i}-\left\langle t_{i}\right\rangle-\rho\right)} \geq 1$ for all $\alpha \geq 0$, so that

$$
p \leq\left\langle\mathrm{e}^{\alpha\left(t_{i}-\left\langle t_{i}\right\rangle-\rho\right)}\right\rangle \leq \mathrm{e}^{-\alpha \rho} \mathrm{e}^{\frac{1}{2} \alpha^{2} G_{i i}}=\mathrm{e}^{-\frac{\rho^{2}}{2 G_{i i}}}
$$

for $\alpha=\rho / G_{i i}$. The same estimate applies to the probability of an event $t_{i}-\left\langle t_{i}\right\rangle \leq-\rho$, so altogether we have

$$
\operatorname{Prob}_{E}\left\{\left|t_{i}-\left\langle t_{i}\right\rangle\right| \geq \rho\right\} \leq 2 \mathrm{e}^{-\frac{\rho^{2}}{2 G_{i i}}} .
$$

Our estimates on the $t$ field will be complete once we have estimated the average $\left\langle t_{i}\right\rangle$. To do this consider the change of variables $t_{j} \rightarrow t_{j}+\gamma, s_{j} \rightarrow \mathrm{e}^{-\gamma} s_{j}(j \in \Lambda)$. Then if we take the derivative in $\gamma$ of the logarithm of the partition function at $\gamma=0$ we get

$$
h \sum_{j \in \Lambda}\left\langle\sinh t_{j}\right\rangle=1 \text {, }
$$

where 1 is produced from the $\delta$-function: $\delta\left(\mathrm{e}^{-\gamma} \sum s_{j}\right)=\mathrm{e}^{\gamma} \delta\left(\sum s_{j}\right)$. By translation invariance we see that for $h|\Lambda|=1$ we have $\left\langle\sinh t_{i}\right\rangle=1$, so that by Jensen's inequality and (5.4) we have

$$
\mathrm{e}^{\left\langle t_{i}\right\rangle} \leq\left\langle\mathrm{e}^{t_{i}}\right\rangle=2+\left\langle\mathrm{e}^{-t_{i}}\right\rangle \leq 2+\mathrm{e}^{-\left\langle t_{i}\right\rangle} \mathrm{e}^{\frac{1}{2} G_{i i}} .
$$

This gives an upper bound to $\left\langle t_{i}\right\rangle$ :

$$
\left\langle t_{i}\right\rangle \leq 1+\frac{1}{4} G_{i i}
$$


To obtain the lower bound we use $\left\langle\sinh t_{i}\right\rangle \geq 0$ :

$$
\mathrm{e}^{-\left\langle t_{i}\right\rangle} \leq\left\langle\mathrm{e}^{-t_{i}}\right\rangle \leq\left\langle\mathrm{e}^{t_{i}}\right\rangle \leq \mathrm{e}^{\left\langle t_{i}\right\rangle} \mathrm{e}^{\frac{1}{2} G_{i i}}
$$

Hence

$$
-\frac{1}{4} G_{i i} \leq\left\langle t_{i}\right\rangle \leq 1+\frac{1}{4} G_{i i}
$$

This completes our estimates on the $t$ fields and its fluctuations.

\section{Bounds on the $s$ fields.}

Recall that in addition to the $t$ variables the observable given by (3.5) contains factors of $s_{0}^{2}$ and $s_{0}^{4}$. These averages may be explicitly calculated in terms of the covariance for the $s$ field given, see (4.1), by $D^{-1}$ on the orthogonal complement of the constant functions which we have denoted by $\widetilde{D}^{-1}$. Here we show how to deal with the $s_{0}^{4}$ term:

$$
\left\langle s_{0}^{4}\right\rangle_{s}=3 \widetilde{D}^{-1}(0,0)^{2}
$$

The $s_{0}^{2}$ term is similar and can be handled in the same way.

The operator $D$ is non-negative but depends on $t$. If all $t_{j} \geq 0$ then $D \geq-\Delta_{\Lambda}$ and we have bounds on $D^{-1}$ in terms of the free Green's function $-\Delta_{\Lambda}^{-1}$ in dimension three. However, the $t$ field may take large negative values and so there is no uniform bound on $\widetilde{D}^{-1}(t)$. The control of $\left\langle\widetilde{D}^{-1}(0,0)^{2}\right\rangle$ will come from the fact that large negative values of $t$ are very rare by (5.5) and (5.7).

To bound the average of $\widetilde{D}^{-1}(0,0)^{2}$ we shall first consider an elliptic operator $L$ whose quadratic form is

$$
(f, L f) \equiv \sum_{j \in \Lambda}(\nabla f)_{j}^{2} a_{j}
$$

where $a_{j} \geq(1+|j|)^{-p}$. Let $\widetilde{L}$ denote the projection on the orthogonal complement of the constant functions.

Lemma 6.1. - For $d \geq 3$ and $p<d-2$ the Green's function of $\widetilde{L}$ is uniformly bounded $\left(\right.$ as $\left.\Lambda \rightarrow \mathbb{Z}^{d}\right)$ by $0 \leq \widetilde{L}^{-1}(0,0) \leq A_{p}<\infty$.

Proof. - Let $C_{n}$ denote the cube of side $2^{n}$ centered at the origin and let $\chi_{n}$ be its indicator function. Note that

$$
f_{n}=2^{-d n} \chi_{n}-2^{-d(n+1)} \chi_{n+1}
$$

has zero average and the square of its $L^{2}$ norm is bounded by $2^{-d n}$.

As a quadratic form, $\widetilde{L}$ restricted to $C_{n}$ (with Neumann boundary conditions) is at least $2^{-(2+p) n}$, and we therefore have

$$
\left(f_{n-1}, \widetilde{L}^{-1} f_{n-1}\right) \leq 2^{(2+p) n} 2^{-d(n-1)} .
$$


To complete the proof of the lemma note that the projection of $\delta_{0}$ onto the orthogonal complement of the constants can be written as a sum over the $f_{n}$. By the Schwarz inequality we have

$$
\widetilde{L}^{-1}(0,0) \leq\left(\sum_{n=0}^{\infty}\left(f_{n}, \widetilde{L}^{-1} f_{n}\right)^{1 / 2}\right)^{2} \leq A_{p}
$$

provided that $p<d-2$.

Lemma 6.2. - There is a constant $c_{0}$ so that

$$
\left\langle\widetilde{D}^{-1}(0,0)^{2}\right\rangle \leq c_{0}
$$

Proof. - Fix some value of $p$ with $0<p \leq \frac{1}{2}$, and for each integer $k$ let $\chi_{k}(t)$ denote the characteristic function of the set of configurations $t=\left\{t_{j}\right\}_{j \in \Lambda}$ that satisfy

$$
\mathrm{e}^{t_{x}+t_{y}} \geq \mathrm{e}^{-k}(|x|+1)^{-p}
$$

for all nearest neighbors $x, y$. We then have $\chi_{k}(t) \widetilde{D}^{-1}(0,0)^{2} \leq\left(A \mathrm{e}^{k}\right)^{2}$ by Lemma 6.1

We now claim that for all $k \geq \kappa \equiv \max \left\{0,-\left\langle t_{x}+t_{y}\right\rangle\right\}$,

$$
\left\langle 1-\chi_{k}(t)\right\rangle_{t} \leq B \mathrm{e}^{-c(k-\kappa)^{2}},
$$

which by Borel-Cantelli gives the desired statement:

$$
\begin{aligned}
\left\langle\widetilde{D}^{-1}(0,0)^{2}\right\rangle & =\left\langle\left(\chi_{0}+\chi_{1}\left(1-\chi_{0}\right)+\chi_{2}\left(1-\chi_{1}\right)+\ldots\right) \widetilde{D}^{-1}(0,0)^{2}\right\rangle \\
& \leq \text { const }+A^{2} B \sum_{k \geq \kappa} \mathrm{e}^{2(k+1)} \mathrm{e}^{-c(k-\kappa)^{2}} \leq \text { const } .
\end{aligned}
$$

To establish our claim 6.3 suppose that

$$
\mathrm{e}^{t_{x}+t_{y}} \leq \frac{\mathrm{e}^{-k}}{(|x|+1)^{p}} \leq \mathrm{e}^{-(k+n p)}
$$

for $x$ in the range $\mathrm{e}^{n} \leq|x| \leq \mathrm{e}^{n+1}$. Then by (5.5) and (5.7) the probability of this event for $k \geq \kappa$ is less than

$$
\mathrm{e}^{(n+1) d} \mathrm{e}^{-c(k-\kappa+n p)^{2}}
$$

whose sum over $n$ is no greater than $\mathrm{e}^{-c(k-\kappa)^{2}}$ times a constant $B$.

The lemma works for any power of $\widetilde{D}^{-1}(0,0)$. Thus by the Schwarz inequality and (5.4) we can bound the expectation of our observable $\mathrm{e}^{2 t_{0}} s_{0}^{4}$. 


\section{Adjusting the regularization}

We have used the $\delta$-function regularization in the $s$ variables rather than the correct term $h \sum_{j \in \Lambda} s_{j}^{2} \mathrm{e}^{t_{j}}$ which appears in the action $A_{\Lambda}$.

Recall $C_{h}=\frac{1}{2} \ln \operatorname{Det}\left(D+h \mathrm{e}^{t}\right)$ and $\widetilde{C}=\frac{1}{2} \ln \operatorname{Det} \widetilde{D}$. We shall express $C_{h}$ in terms of $\widetilde{C}$. To do this, let $P_{0}$ denote the orthogonal projector on the vector space spanned by the normalized constant function $\psi_{0, j}=|\Lambda|^{-1 / 2}$, and let $P=1-P_{0}$. The determinant can be calculated in terms of $P$ and $P_{0}$ blocks:

$$
\operatorname{Det}\left(D+h \mathrm{e}^{t}\right)=\operatorname{Det}\left(\widetilde{D}+h P_{t}\right)\left(\psi_{0}, h \mathrm{e}^{t} \psi_{0}\right),
$$

where $P_{t}$ is given by

$$
P_{t}=P \mathrm{e}^{t} P-P \mathrm{e}^{t} P_{0} \mathrm{e}^{t} P \cdot\left(\psi_{0}, \mathrm{e}^{t} \psi_{0}\right)^{-1} .
$$

Using the Schwarz inequality it is easy to see that $P_{t} \geq 0$.

Thus $C_{h}=\widetilde{C}+\frac{1}{2} \operatorname{Tr} \ln \left(1+h \widetilde{D}^{-1} P_{t}\right)+\frac{1}{2} \ln \left(\psi_{0}, \mathrm{e}^{t} \psi_{0}\right)$. We have left out the $\ln h$ term since it is cancelled in the normalization.

Let $F(t)$ be our (positive) observable. Now we can write

$$
\langle F(t)\rangle_{E_{h}}=\frac{\left\langle F(t) \mathrm{e}^{-R_{h}}\right\rangle_{E}}{\left\langle\mathrm{e}^{-R_{h}}\right\rangle_{E}}
$$

where

$$
R_{h}=C_{h}-\widetilde{C}=\frac{1}{2} \operatorname{Tr} \ln \left(1+h \widetilde{D}^{-1} P_{t}\right)+\frac{1}{2} \ln \left(\psi_{0}, \mathrm{e}^{t} \psi_{0}\right) .
$$

Since the first term of $R_{h}$ is positive we have

$$
\langle F(t)\rangle_{E_{h}} \leq \frac{\left\langle F(t)\left(\psi_{0}, \mathrm{e}^{t} \psi_{0}\right)^{-\frac{1}{2}}\right\rangle_{E}}{\mathrm{e}^{-\left\langle R_{h}\right\rangle_{E}}} \leq\left\langle F(t) \mathrm{e}^{-\frac{1}{2}\left(\psi_{0}, t \psi_{0}\right)}\right\rangle_{E} \mathrm{e}^{\left\langle R_{h}\right\rangle_{E}}
$$

where we have used Jensen's inequality. Since $P_{t}$ is positive we have

$$
\left\langle\operatorname{Tr} \ln \left(1+h \widetilde{D}^{-1} P_{t}\right)\right\rangle_{E} \leq h\left\langle\operatorname{Tr}\left(\widetilde{D}^{-1} P_{t}\right)\right\rangle_{E} \leq h|\Lambda|\left\langle\mathrm{e}^{t_{0}} \widetilde{D}^{-1}(0,0)\right\rangle_{E} \leq \mathrm{const},
$$

and the other term in $\left\langle R_{h}\right\rangle$ is estimated by

$$
\left\langle\frac{1}{2} \ln \left(\psi_{0}, \mathrm{e}^{t} \psi_{0}\right)\right\rangle \leq \frac{1}{2}\left\langle\mathrm{e}^{t_{0}}\right\rangle \leq \text { const } .
$$

The desired bound on $\langle F\rangle_{E_{h}}$ now follows from estimates we obtained for \langle\rangle$_{E}$. This completes our proof of Theorem 1.1

\section{Appendix: Push forward of measure in Fyodorov's method}

Consider the mapping

$$
\psi: \operatorname{Hom}\left(\mathbb{C}^{n}, \mathbb{C}^{N}\right) \rightarrow \operatorname{Herm}^{+}\left(\mathbb{C}^{n}\right), \quad \varphi \mapsto \varphi^{*} \varphi=M,
$$


and fix some (translation-invariant) Lebesgue measure $d \varphi d \bar{\varphi}$ on $\operatorname{Hom}\left(\mathbb{C}^{n}, \mathbb{C}^{N}\right)$. We claim that, if $N \geq n$, there exists a Lebesgue measure $c_{n, N} d M=d M_{n, N}$ (with normalization constant depending on $n$ and $N$ ) such that the equality

$$
\int_{\operatorname{Hom}\left(\mathbb{C}^{n}, \mathbb{C}^{N}\right)} F\left(\varphi^{*} \varphi\right) d \varphi d \bar{\varphi}=\int_{\operatorname{Herm}^{+}\left(\mathbb{C}^{n}\right)} F(M) \operatorname{Det}^{N-n}(M) d M_{n, N}
$$

holds for all functions $M \mapsto F(M)$ on $\operatorname{Herm}^{+}\left(\mathbb{C}^{n}\right)$ with finite integral $\int F\left(\varphi^{*} \varphi\right) d \varphi d \bar{\varphi}$. In other words, $\psi$ pushes the measure $d \varphi d \bar{\varphi}$ forward to

$$
\psi(d \varphi d \bar{\varphi})=\operatorname{Det}^{N-n}(M) d M_{n, N} .
$$

While this claim can be viewed and proved as a statement in invariant theory, the most elementary proof is to express the integrals on both sides in terms of generalized polar coordinates, as follows.

Given any complex rectangular matrix $\varphi \in \operatorname{Hom}\left(\mathbb{C}^{n}, \mathbb{C}^{N}\right)$ for $N \geq n$, consider the non-negative Hermitian matrices $M=\varphi^{*} \varphi$ and $M^{\prime}=\varphi \varphi^{*}$, which are of size $n \times n$ and $N \times N$ respectively. The rank of $M^{\prime}$ cannot exceed $n$, so there must be at least $N-n$ zero eigenvalues. The other $n$ eigenvalues are in general non-zero, and coincide with the eigenvalues of $M=\varphi^{*} \varphi$. Denote these eigenvalues by $\lambda_{1}, \ldots, \lambda_{n}$; their positive square roots $\sqrt{\lambda_{k}}$ are sometimes called the singular values of $\varphi$. There always exist two unitary matrices $U \in \mathrm{U}(n)$ and $V \in \mathrm{U}(N)$ such that

$$
\varphi^{*}=U \sqrt{\lambda}^{\mathrm{T}} V^{-1}, \quad \varphi=V \sqrt{\lambda} U^{-1},
$$

where $\sqrt{\lambda}$ is the rectangular $N \times n$ matrix with diagonal entries $\sqrt{\lambda_{1}}, \ldots, \sqrt{\lambda_{n}}$ and zeroes everywhere else.

Let $J(\sqrt{\lambda})$ be the Jacobian of this singular value (or polar) decomposition:

$$
J(\sqrt{\lambda})=\prod_{1 \leq i<i^{\prime} \leq n}\left(\sqrt{\lambda_{i}}-\sqrt{\lambda_{i^{\prime}}}\right)^{2}\left(\sqrt{\lambda_{i}}+\sqrt{\lambda_{i^{\prime}}}\right)^{2} \prod_{k=1}^{n}{\sqrt{\lambda_{k}}}^{1+2(N-n)} .
$$

Fix the values of $n$ and $N \geq n$. Then by a standard argument there exists some (fixed) choice of Haar measure $d U$ for $\mathrm{U}(n)$ such that

$$
\int_{\operatorname{Hom}\left(\mathbb{C}^{n}, \mathbb{C}^{N}\right)} F\left(\varphi^{*} \varphi\right) d \varphi d \bar{\varphi}=\int_{\mathbb{R}_{+}^{n}}\left(\int_{\mathrm{U}(n)} F\left(U \lambda U^{-1}\right) d U\right) J(\sqrt{\lambda}) \prod_{k=1}^{n} d \sqrt{\lambda_{k}}
$$

holds for all integrable $F$. Here $\lambda=\operatorname{diag}\left(\lambda_{1}, \lambda_{2}, \ldots, \lambda_{n}\right)$.

On the other hand, fix some Lebesgue measure $d M$ for $\operatorname{Herm}\left(\mathbb{C}^{n}\right)$. By diagonalizing the Hermitian matrix $M$ and transforming the integral $\int f(M) d M$ to the eigenvalue representation $M=U \lambda U^{-1}$ you get

$$
\int_{\mathrm{Herm}^{+}\left(\mathbb{C}^{n}\right)} f(M) d M=b_{n, N} \int_{\mathbb{R}_{+}^{n}}\left(\int_{\mathrm{U}(n)} f\left(U \lambda U^{-1}\right) d U\right) \prod_{i<j}\left(\lambda_{i}-\lambda_{j}\right)^{2} \prod_{k} d \lambda_{k} .
$$


The constant $b_{n, N}$ is determined by the (arbitrary) choice of $d M$ relative to $d U$. Now put $f(M)=\operatorname{Det}^{N-n}(M) F(M)$. Since $\operatorname{Det}^{N-n}(M)=\prod_{k} \lambda_{k}^{N-n}$, the desired statement (8.1) follows (with $c_{n, N}=2^{-n} / b_{n, N}$ ) by comparing expressions and noting

$$
\prod_{i<j}\left(\lambda_{i}-\lambda_{j}\right)^{2} \prod_{k} \lambda_{k}^{N-n} d \lambda_{k}=2^{n} J(\sqrt{\lambda}) \prod_{k} d \sqrt{\lambda_{k}}
$$

The relation 8.1 can also be viewed from another perspective, which we shall now offer. First note that the integral on the left-hand side can be regarded as a distribution (or continuous linear functional), say $\mu$, on $\operatorname{Herm}^{+}\left(\mathbb{C}^{n}\right)$ :

$$
\mu: F \mapsto \int F\left(\varphi^{*} \varphi\right) d \varphi d \bar{\varphi}
$$

Next observe that the non-compact Lie group $\operatorname{GL}(n, \mathbb{C})$ acts transitively on the positive Hermitian $n \times n$ matrices $M$ by

$$
M \mapsto T M T^{*} \quad(T \in \mathrm{GL}(n, \mathbb{C})) .
$$

Via this action we can identify (a dense open subset of) $\mathrm{Herm}^{+}\left(\mathbb{C}^{n}\right)$ with the noncompact symmetric space $\mathrm{GL}(n, \mathbb{C}) / \mathrm{U}(n)$. The corresponding action on functions, $F \mapsto{ }^{T} F$, is given by

$$
{ }^{T} F(M)=F\left(T^{-1} M T^{-1^{*}}\right) .
$$

Given that $T \in \operatorname{GL}(n, \mathbb{C})$ acts on the functions, there is also an action $\mu \mapsto T(\mu)$ on the distributions, by $T(\mu)[F]=\mu\left[T^{-1} F\right]$. Since the Jacobian of the transformation $\varphi \mapsto$ $\varphi \circ T^{*}$ and $\varphi^{*} \mapsto T \circ \varphi^{*}$ is $\operatorname{Det}^{N}\left(T^{*} T\right)$, the distribution $\mu$ at hand satisfies

$$
T(\mu)=\operatorname{Det}^{N}\left(T^{*} T\right) \mu .
$$

Now we make the same considerations on the right-hand side of (8.1), i.e. for the distribution

$$
\widetilde{\mu}: F \mapsto \int F(M) \operatorname{Det}^{N-n}(M) d M
$$

Under the transformation $M \mapsto T M T^{*}$ the Lebesgue measure $d M$ transforms into $\operatorname{Det}^{n}\left(T^{*} T\right) d M$. Hence $\operatorname{Det}^{-n}(M) d M$ is invariant under such transformations, and

$$
T(\widetilde{\mu})=\operatorname{Det}^{N}\left(T^{*} T\right) \widetilde{\mu},
$$

i.e., $\widetilde{\mu}$ transforms in exactly the same way as $\mu$. It is an invariant-theoretic fact resulting from the interpretation of $\mu$ as a linear functional on $\mathrm{U}(N)$-invariant state vectors $F$ in the oscillator or Shale-Weil representation of $\operatorname{Sp}(2 n N)$ - that the vector space of distributions with this transformation property has dimension one. Therefore, there exists some constant $c_{n, N}$ such that

$$
\mu=c_{n, N} \times \widetilde{\mu} .
$$

As a corollary, we note that $\operatorname{Det}^{-n}(M) d M$ is an invariant measure for the symmetric space of positive Hermitian $n \times n$ matrices, $\operatorname{Herm}^{+}\left(\mathbb{C}^{n}\right) \simeq \mathrm{GL}(n, \mathbb{C}) / \mathrm{U}(n)$. The case encountered in the main text of the paper is obtained by replacing $n \rightarrow 2 n$. 
T. SPENCER \& M.R. ZIRNBAUER

\section{References}

[B] Brascamp, H. and Lieb, E.: On extensions of the Brunn-Minkowski and Prekopa-Leindler theorems, including inequalities for log concave functions, and with an application to the diffusion equation, J. Func. Anal. 22, 366-389 (1976)

[D] Disertori, M., Pinson, H. and Spencer, T.: Density of states of random band matrices, Commun. Math. Phys. 232, 83-124 (2002)

[E] Efetov, K.B.: Supersymmetry and theory of disordered metals, Adv. Phys. 32, 53-127 (1983)

[E1] Efetov, K.B.: Supersymmetry in disorder and chaos (Cambridge University Press, 1987)

[F] Fyodorov, Y.V.: Negative moments of characteristic polynomials of random matrices: Ingham-Siegel integral as an alternative to Hubbard-Stratonovich transformation, Nucl. Phys. B 621, 643-674 (2002)

[FM] Fyodorov, Y.V. and Mirlin, A.D.: Scaling properties of localization in random band matrices: a б-model approach, Phys. Rev. Lett. 67, 2405-2409 (1991)

$[\mathrm{H}]$ Helgason, S.: Differential geometry, Lie groups and symmetric spaces (Academic Press, New York, 1978)

[N] Niedermaier, M. and Seiler, E.: Non-amenability and spontaneous symmetry breaking - the hyperbolic spin-chain, arXiv:hep-th/0312293; Duncan, A., Niedermaier, M. and Seiler, E.: Vacuum orbit and spontaneous symmetry breaking in hyperbolic sigma models, arXiv:hep-th/0405163

[S] Schäfer, L. and Wegner, F.: Disordered system with $n$ orbitals per site: Lagrange formulation, hyperbolic symmetry, and Goldstone modes, Z. Phys. B 38, 113-126 (1980)

[W] Wegner, F.: The mobility edge problem: continuous symmetry and a conjecture, Z. Phys. B 35, 207-210 (1979)

[W1] Wegner, F.J.: Disordered system with $n$ orbitals per site: $n=\infty$ limit, Phys. Rev. B 19, 783-792 (1979)

[Z] Zirnbauer, M.R.: The supersymmetry method of random-matrix theory, arXiv:math-ph/ 0404057

September 21, 2004

T. SPEnCER, School of Mathematics, Institute for Advanced Study, Princeton N.J., USA

M.R. ZIRnBAUER, Institut für Theoretische Physik, Universität zu Köln, Germany

E-mail: spencer@math.ias.edu, zirn@thp.uni-koeln.de 